\title{
“Actividades realizadas para la organización de Clínicas para el manejo del Climaterio en Colombia” y Latinoamérica
}

\author{
William Onatra H. MD.*
}

\begin{abstract}
RESUMEN: Se presenta en forma cronológica el desarrollo de las actividades de un grupo de personas del área de la salud deseosos de estudiar y colocar su conocimiento al servicio de la mujer durante este período y que desemboca en la organización de la Asociación Colombiana de Menopausia.
\end{abstract}

SUMMARY: In a chronological way we present the development of the activities made by a group of people from health area wanting to study and give their knowledge to woman's service during this period of life resulting in the organization of Menopause Colombian Association

Con el descubrimiento del origen y síntesis de los estrógenos por Allan y Doysi en 1923 y la utilidad durante la postmenopausia propuesta por investigadores tanto europeos como americanos, en la década del 40-50, abrieron una nueva perspectiva terapéutica para este grupo etario, publicándose a nivel mundial sus riesgos beneficios (1).

En nuestro medio fue sólo en la década de los 80 cuando se incluye el tema de la Menopausia en los programas de pregrado de las Facultades de Medicina. Un análisis de los textos de Medicina Interna demuestra que el tema es mencionado, pero no se presenta en la orientación y manejo que se le da hoy al Climaterio (2). Para este momento se inicia en forma tímida la consulta externa de Menopausia en el Hospital San José a cargo del Dr. Roberto Jaramillo U, Hospital Lorencita Villegas de Santos con el Dr. Jaime Urdinola y en el Hospital Materno Infantil el Dr. William Onatra H. (Octubre 10 de 1987). Los Drs. Israel Díaz y Edgar Mesa Díaz-Granados de Barranquilla ya eran miembros de la Sociedad América de Menopausia. Para este último quinquenio tanto ASMEDAS Nacional como Cundinamarca incluyen en sus programas de educación continua el tema de «Manejo de la mujer menopáusica». Con la introducción de la vía transdérmica entre los años 1990-91 se realizan una serie de conferencias sobre el diagnóstico y manejo de la mujer climatérica auspiciadas por la Federación Colombiana de Obstetricia y Ginecología (FECOLSOC), en todo el país.

- Congreso Latinoamericano de Montevideo, noviembre 91 , se realiza una reunión con los representantes de Argentina, Brasil, Venezuela, Chile, Uruguay, Paraguay,

* Profesor Asistente Departamento de Obstetricia y Ginecología. Facultad de Medicina. Universidad Nacional de Colombia. Clínica de Menopausia. Instituto Materno Infantil. Bogotá. Presidente Asociación Colombiana de Menopausia.
México y se firma un acta para la conformación de la Sociedad Latinoamericana de Climaterio y Menopausia (FLASCYM), en representación de Colombia asisten los Drs. R. Jaramillo y W. Onatra.

Durante la celebración del XVIII Congreso Colombiano de Obstetricia y Ginecología el día 6 de diciembre de 1991 en Barranquilla se lleva a cabo un desayuno de trabajo en el Hotel Caribe aprovechando la visita del Dr. Santiago Palacios G. de España, secretario de la Asociación Europea de Menopausia, quien deseaba la organización de estudios a nivel Latinoamericano. Con la representación de los siguientes Ginecólogos del país, se conforma el «Primer Grupo para el estudio del Climaterio: Barranquilla: Drs. Israel Díaz R, Edgar Mesa Díaz Granados, Magali Valera O, Alvaro Anillo M.

Bogotá: Francisco Pardo V, Roberto Jaramillo U, Jaime

Urdinola, Luis E. Pérez, Gabriel Acuña A, Clara

Zambrano de R, Rocío Fuquen J, José Ignacio Madero, José María Fuentes, Jacinto Sánchez A, Ricardo Alvarado.

Bucaramanga: Hermes Jaimes C.

Cali: José Zuñiga G, Gustavo Gómez T.

Cartagena: Antonio Soto Y, Marco Yances O, Jaime Barrios A, Alvaro Monterrosa, Fabio Ramírez, Andrés López M.

Manizales: Hernán Giraldo E.

Medellín: Fabio Sánchez, Aníbal Castañeda.

Ibagué: Jaime Rengifo A.

Valledupar: Jorge Rugeles, Ana R. Cortés.

Con el propósito de llevar a cabo un estudio epidemiológico multicéntrico, se nombran representantes en cada ciudad, en los centros Hospitalarios del país y por deseo del grupo se elige como coordinador del estudio al Dr. W. Onatra H. Se solicita presentar en la asamblea de FECOLSOC que el tema «Climaterio» sea tenido en cuenta dentro de las ponencias para el próximo Congre- 
so de Obstetricia y Ginecología que se llevaría a cabo en Santafé de Bogotá en 1993, propuesta que fue acogida por unanimidad.

Para diciembre 30 del 91 se diseña el protocolo epidemiológico, el formulario de recolección de datos y se busca el apoyo estadístico en la Unidad Epidemiológica de la Universidad Javeriana de Bogotá.

Durante el año de 1992 se continúan dictando conferencias con el auspicio de las Sociedades Científicas de Obstetricia y Ginecología, con el apoyo de la industria farmacéutica se lleva una actualización sobre el manejo integral del climaterio a Medellín, Cali, Barranquilla, Cartagena, Valledupar, Montería, Pereira, Bucaramanga, Neiva, Buga y Popayán.

Gracias al entusiasmo del Dr. Fabio Sánchez y de los residentes de Gineco-Obstetricia del Hospital Universitario de Antioquia se realiza el primer Curso Nacional sobre el Climaterio en la ciudad de Medellín editándose las conferencias dictadas en marzo de 1992.

- Conformación de la Asociación de Menopausia del Atlántico y primer Boletín informativo sobre el Climaterio:

Bajo la dirección de los doctores Israel Díaz y el Dr. Edgar Meza se conforma la Asociación de Menopausia del Atlántico «ADMAT», iniciando actividades en 1992 produciendo el primer boletín de la Asociación que en forma ininterrumpida se ha mantenido durante los años 1993 y 1994. Realizaron el I Simposio sobre menopausia en la costa en agosto de 1993 con notable éxito. Actualmente es uno de los grupos más activos con una mesa directiva muy dinámica llevando a cabo varias reuniones mensuales sobre el tema y programaron su II curso regional para agosto 12 y 13 de 1994 .

En octubre de 1992 en la ciudad de Cartagena, durante la celebración de los 25 años de FECOLSOG, se presenta la orientación que se está llevando a cabo en el país en las Clínicas de Climaterio y la experiencia en una clínica de Bogotá.

Se asiste al II Congreso Europeo de Menopausia (Octubre 29 a 31 de 1992) celebrado en Madrid - España, entregando las memorias a diferentes coordinadores.

Para 1993 durante el IV Curso bienal de la Sociedad Colombiana de Obstetricia y Ginecología, realizado en el mes de marzo de 1993, se dedica una mañana al tema del climaterio. En la ciudad de Cali con la coordinación del Dr. Gustavo Gómez y los Residentes de GinecoObstetricia del Hospital Universitario del Valle, se lleva a cabo el II Curso Nacional sobre climaterio durante los días 7 y 8 de mayo del 93, dejando también editados sus temas.

La Asociación de Menopausia del Atlántico programa su I curso Regional en agosto del 93 con la participación de diferentes universidades del país. En la sesión final en noviembre de 1993, de la Sociedad Vallecaucana, se incluye el manejo actual de la terapia hormonal de sustitución con activa participación de sus delegados.

- Durante el Congreso Mundial de Infertilidad celebrado en Caracas se consolida la directiva Latinoamericana para la organización de las asociaciones para el estudio del Climaterio y Menopausia FLASCYM, en representación de Colombia asisten los Drs. J. Urdinola, Ignacio Madero y W. Onatra H.

Actividades a la comunidad: Durante estos dos años en algunas ciudades se dictaron conferencias tanto por los coordinadores de área como por otros especialistas información a la comunidad sobre el tema del climaterio. Se hace difusión en programas radiales, televisivos y algunas revistas de promoción de salud.

En el Congreso Latinoamericano celebrado en Panamá en el tema de Menopausia representan a Colombia como conferencista Dr. F. Pardo, J. Urdinola y W. Onatra. Se realiza la Asamblea de la FLASCYM con representación de los Drs. F. Pardo y W. Onatra y se plantea la necesidad de organizar la asociación en Colombia. Se invita a todos los países miembros al I curso latinoamericano que se celebrará en Buenos Aires Octubre del 95.

Durante el XIX Congreso Colombiano de Obstetricia y Ginecología celebrado en la ciudad de Santafé de Bogotá el día 3 de marzo en el Centro de Convenciones Gonzalo Jiménez de Quesada se presenta el estudio multicéntrico cooperativo sobre algunas características epidemiológicas de la mujer climatérica en una población en su mayoría hospitalaria colombiana con la participación de los siguientes centros:

Hospital San Vicente de Paul, Medellín, Dr. F. Sánchez

Asociación de Menopausia del Atlántico, Barranquilla, Dr. I. Díaz Dr. E. Meza DG.

Hospital Ramón González V. Bucaramanga, Dr. H. Jaimes

Hospital Universitario del V. Cali, Dr. G. Gómez. T. Santafé de Bogotá

Hospital San José, Dr. R. Jaramillo U, A Ruiz

Hospital Lorencita V. de Santos, Dr. Barón

Hospital Militar Central, Dr. J. de la Cruz

Hospital San Ignacio, Dr. F. Pardo V.

Hospital San Rafael, Dr. G.D Arango P.

Instituto de Climaterio. Dr. G. Acuña .A.

Instituto de Seguros Sociales, Dr. G. Tovar

Instituto Materno Infantil, Dr. J. Sánchez. A

Los resultados del estudio están presentados en el suplemento del Vol 45 No. 4/94 de la Revista Colombiana de Obstetricia y Ginecología.

Durante este evento el día 3 de marzo de 1994, con el auspicio de las directivas de la Sociedad Colombiana de Obstetricia y Ginecología se decide organizar la Asociación Colombiana de Menopausia, con representación de diferentes delegados del país, eligiendo como presidente al Dr. William Onatra, Vicepresidente el Dr. Edgar Meza, Secretario el Dr. Jaime Urdinola, Tesorero, el Dr. Gabriel Acuña. Vocales a los Dres. Israel Díaz de Barranquilla, Dr. Fabio Sánchez de Medellín, Dr. Gustavo Gómez de Cali y Hermes Jaimes de Bucaramanga, quedando pendiente la representación de Bogotá. Se entrega un proyecto de estatutos a los asistentes para se estudiado y modificado en cada uno de los capítulos.

El 21 de junio de 1994 en la Clínica David Restrepo se conforma el Capítulo de Bogotá, siendo elegido Presidente el Dr. Roberto Jaramillo, Secretario Rosmery Díaz del Castillo; Tesorero Dr. Alfredo Ruiz y como Vocales los diferentes representantes de los Hospitales en Bogotá así: 
HLV Dr. Germán Barón

Hospital San Rafael

Materno Infantil

San Blas

San José

Samaritana

ISS

San Ignacio

Clínica de la Policía

Oriéntame

El 22 de agosto/94 se conforma el Capítulo de Medellín con elección para la Presidencia el Dr. Fabio Sánchez, Vicepresidente, Antonio José Restrepo, Secretaria, Stella Moreno Vélez, Tesorero, Alfonso Jubiz, Vocales los diferentes miembros de la comunidad médica.

El 8 de Septiembre/94 se conforma el Capítulo de Bucaramanga con la Presidencia del Dr. Hermes Jaimes, Vicepresidente, Reynaldo Badillo, Secretaria, Elizabeth Herrera, Tesorero, Luz Amparo Ledezma y vocales los representantes de la comunidad.
Se encuentran pendientes Cúcuta, Cartagena y el eje Cafetero.

En septiembre de 1994 se programa una teleconferencia desde Atlanta en la ciudad de Santafé de Bogotá, sobre la utilidad del tratamiento cíclico o continuo, dictada por el Dr. R. Waiss, con participación de los coordinadores del programa epidemiológico.

El 3 de noviembre/94 se conforma el capítulo del Valle con la siguiente Junta Directiva: Presidente, Gustavo Gómez Tabares, Vicepresidente, Dra. María Cecilia Arturo R., Secretario, Libardo Bravo, Tesorero, Luis Fernando Medina.

Se ha dado pues nacimiento a este equipo de trabajo, con participación de Enfermeras, Psicólogos, Terapistas, Nutricionistas, Trabajadores Sociales y todas las especialidades de la medicina con el fin de integrar a la mujer en uno de sus períodos críticos pero más maravillosos de su existencia.

\section{BIBLIOGRAFIA}

1. Wilbush J. Climateric Disorders -Historical perspective. in The Menopause Studd JWW.Blaclwell Scie.Publi.Oxford 1988:1-14

2. Hemminki E., Sihvo S.A review of postmenopausal hormone therapy recommendationes: potential for selection bias. Obstet Gynecol $1993 ; 82: 1021-1028$
3. Dawood MY, Tidey GF. Menopause. Curr Probl Obstet Gynecol Fertil 1993;16:169-208 